\title{
HIV/AIDS-Related Institutional Mistrust among Multi-Ethnic Men Who Have Sex with Men: Effects on HIV Testing and Risk Behaviors
}

\author{
Michael A. Hoyt \\ University of California, Merced \\ Lisa R. Rubin \\ New School for Social Research \\ Carol J. Nemeroff \\ University of Southern Maine \\ Joyce Lee \\ University of Minnesota \\ David M. Huebner \\ University of Utah \\ Rae Jean Proeschold-Bell \\ Duke University
}

Michael A. Hoyt is now at Department of Psychology, City University of New York, Hunter College.

Correspondence concerning this article should be addressed to Michael A. Hoyt, City University of New York, Hunter College, Department of Psychology, 695 Park Avenue, Room 611-HN, New York, NY 10065. E-mail:

mho0015@hunter.cuny.edu

This research was supported in part by a grant from the Centers for Disease Control and Prevention to the Arizona Department of Health Services through Cooperative

Agreement 99004.

\section{Abstract}

Objective: To investigate relationships between institutional mistrust (systematic discrimination, organizational suspicion, and conspiracy beliefs), HIV risk behaviors, and HIV testing in a multi-ethnic sample of men who have sex with men (MSM), and to test whether perceived susceptibility to HIV mediates these relationships for White and ethnic minority MSM.

Methods: Participants were $394 \mathrm{MSM}$ residing in Central Arizona ( $M$ age = 37 years). Three dimensions of mistrust were examined including Organizational Suspicion, Conspiracy Beliefs, and Systematic Discrimination. Assessments of sexual risk behavior, HIV testing, and perceived susceptibility to HIV were made at study entry (T1) and again six months later (T2).

Results: There were no main effects of institutional mistrust dimensions or ethnic minority status on T2 risk behavior, but the interaction of Systematic Discrimination and Conspiracy Beliefs with minority status was significant such that higher levels of Systematic Discrimination and more Conspiracy Beliefs were associated with increased risk only among ethnic minority MSM. Higher levels of Systematic Discrimination was significantly related to lower likelihood for HIV testing, and the interaction of Organizational Suspicion with minority status was significant such that greater levels of Organizational Suspicion was related to less likelihood of having been tested for HIV among ethnic minority MSM. Perceived susceptibility did not mediate these relationships.

Conclusion: Findings suggest that it is important to look further into the differential effects of institutional mistrust across marginalized groups, including sexual and ethnic minorities. Aspects of mistrust should be addressed in HIV prevention and counseling efforts.

Key Words: Institutional Mistrust, Conspiracy Beliefs, Sexual Risk Behavior, HIV/AIDS

This is an author-produced PDF of an article accepted for publication in the journal Health Psychology following peer review. The full article citation is: Hoyt, M. A., Rubin, L., Nemeroff, C. J., Lee, J., Huebner, D. M., \& Proeschold-Bell, R. J. (2012). HIV/AIDS-related institutional mistrust among multi-ethnic men who have sex with men: Effects on HIV testing and risk behaviors. Health Psychology, 31(3), 269-277. DOI: 10.1037/a0025953. The final publication is available at: http://psycnet.apa.org/journals/hea/31/3/269/. This article was published Online First November 7, 2011. 


\section{Introduction}

Mistrust in medical institutions is associated with important health behaviors, including utilization of health care services (e.g., Musa, Sculz, Harris, Silverman, \& Thomas, 2009), adherence to prevention and treatment recommendations (e.g., Bogart, Wagner, Galvan, \& Banks, 2010), participation in biomedical research (e.g., Scharff, Mathews, Hoffsuemmer, Martin, \& Edwards, 2010), and engagement in health risk behavior (e.g., Ross, Essian, \& Torres, 2006). Institutional mistrust develops when individuals believe their interests are not aligned with the priorities or perceived motives of established institutions (Omodei \& McLennan, 2000). As a result, the fairness and intentions of institutions may be questioned, perceived as discriminative, or construed as harmful or malicious. Further, marginalization from the dominant culture is likely to foster greater mistrust towards institutions perceived to be constructed by and/or positioned for the benefit of the majority. Institutional mistrust is especially important in the context of HIV risk behaviors, particularly for members of sexual and ethnic minority groups, who are generally at heightened risk for HIV infection.

Men who have sex with men (MSM) remain the risk group most seriously affected by HIV. MSM account for nearly half of all individuals living with HIV, and more than half of all new HIV infections, in the U.S. each year (CDC, 2010), with racial/ethnic minority MSM disproportionately affected (CDC, 2007). Part of the ethnic disparity in HIV infection might result from differential access to or utilization of HIV testing and treatment services. For example, it has been documented that Black MSM tend to be less likely to have been tested for HIV in comparison to White and other ethnic minority MSM (Magnus et al., 2010). Moreover, factors related to health care usage (e.g., delayed time to diagnosis, untreated sexually transmitted infection) better explain disparities in HIV risk than risk behavior (Millett, Peterson, Wolitski, \& Stall, 2006).

Although both ethnic minority and White MSM experience marginalization from the dominant culture that can engender feelings of mistrust of medical institutions, the degree or form may differ for these groups as a result of different social histories and experiences (e.g., Malebranche, Peterson, Fullilove, \& Stackhouse, 2004). Thus, understanding institutional mistrust is critical to the success of targeted health promotion efforts aimed at preventing HIV-related risk behaviors and increasing uptake of HIV testing, particularly within marginalized groups.

Mistrust in medical institutions tends to be experienced at higher levels among ethnic (Bird \& Bogart, 2005; Halbert et al., 2009; Thompson, Valdimarsdottir, Winkel, Jandorf, \& Redd, 2004) and sexual (Owens, Riggle, \& Rostosky, 2007) minority groups. In addition to histories of racism and homophobia in medicine (Dean et al., 2000; Smedley, Stih, \& Nelson, 2003), critical occurrences have been linked to the development and promulgation of mistrust in health care institutions. Most notably, the Tuskegee Study of Untreated Syphilis that extended from the early 1930s through the 1970s has been frequently identified as a noteworthy contributor to past and current medical mistrust among African Americans (e.g., Brandon, Isaac, \& LaVeist, 2005; Gamble, 1997). Among Native Americans, tribal histories documenting the distribution of small pox blankets (Vernon \& Jumper-Thurman, 2002), and involuntary sterilization of African American (Roberts, 1997), Puerto Rican (López, 2008), and Mexican

American women (Gutiérrez, 2008) have all been cited as contributing to medical mistrust among diverse ethnic minority groups. 
HIV/AIDS-related mistrust in health care institutions emerged during the first decade of the epidemic, as sexual minority groups, in particular, criticized governmental and non-governmental health care institutions for the lack of progress in and low priority assigned to its research and treatment (Jennings \& Andersen, 2003; Sheran, 1995). Perhaps partly as a result, mistrust and conspiracy beliefs about the origins, prevention, and treatment of HIV/AIDS also formed (see Kalichman, 2009; Whetten, Reif, Whetten, \& Murphy-McMillan, 2008). For instance, research has found that $15 \%-35 \%$ of African Americans believe that AIDS is a form of genocide (Bogart \& Bird, 2003; Bogart \& Thorburn, 2005; Gamble, 1997; Herek \& Capitanio, 1994; Klonoff \& Landrine, 1999; Parsons, Simmons, Shinhoster, \& Kilburn, 1999; Thomas \& Quinn, 1993), and a recent study found that over one-fifth of Latinos in their sample similarly endorsed the belief that AIDS is a form of genocide against minority populations (Ross, Essien, \& Torres, 2006). Other specific conspiracy and mistrust beliefs have been identified. Bogart and Bird (2003) reported that $48 \%$ of African Americans believed that HIV is man-made, and $27 \%$ believed that AIDS was produced in a government laboratory. Endorsing conspiracy beliefs might reasonably be expected to lead to rejection of prevention recommendations or services, particularly if government sponsored.

Some evidence suggests that such mistrust beliefs are also associated with greater HIV risk behavior. In a cross-sectional telephone survey of a random sample of African Americans, Bogart and Thorburn (2005) reported that stronger HIV/AIDS conspiracy beliefs were associated with more negative attitudes and less consistent condom use among men; negative attitudes towards condoms partially mediated the relationship between conspiracy beliefs and condom use. Moreover, Bogart and colleagues (Bogart, Wagner, Galvan, \& Banks, 2010; Bogart, Galvan, Wagner, \& Klein, 2010) have found that HIV-positive African American men have relatively high levels of conspiracy beliefs - with almost two-thirds of their sample endorsing at least one HIV conspiracy belief and almost half endorsing at least two beliefs. They found that endorsement of HIV conspiracy beliefs, particularly treatment-related beliefs (e.g., antiretroviral medication causes AIDS), were related to lower likelihood of optimal treatment adherence and a higher likelihood of unprotected intercourse. The findings are striking, and suggest the need for deeper understanding of the relationships between mistrust and HIV risk-related perceptions and behaviors.

Socialization processes and marginalization have been identified as correlates of medical mistrust and stronger conspiracy beliefs (see Hammond, 2010). However, research examining the influence of mistrust on health behavior, such as the work by Bogart et al. (2010) noted above, has only just begun to elucidate mechanisms by which mistrust might influence health behaviors, and more research is needed to address these questions. For instance, does HIV-related institutional mistrust alter perceptions of vulnerability to HIV which in turn affects engagement in risk or protective behaviors? In fact, well established theories have conceptualized a role for perceived susceptibility in affecting cognitive appraisals of health-related threats (see Mullen, Hersey, \& Iverson, 1987). For instance, the Extended Parallel Processes Model (EPPM; Witte, 1992) suggests that perceived susceptibility to a threat shape the nature of a response to that threat. The question of whether mistrust leads to higher or lower levels of perceived susceptibility remains. Arguably, mistrust in HIV-related institutions might leave one feeling vulnerable to targeted malicious intent and perceptions that one will become an inevitable victim. On the other hand, mistrust has been associated with a disbelief in the existence of HIV or a denial of conventional knowledge regarding HIV transmission (see Kalichman, 2009). Moreover, it is 
possible that the nature of the relationship between mistrust and HIV testing and risk behaviors is different for White and ethnic minority MSM. For example, a qualitative study of multiple minority stress among Black lesbians revealed that participants' experiences of heterosexism and homophobia were often contextualized through a prism of racism (Bowleg, Huang, Brooks, Black, \& Burkholder, 2003). Therefore, institutional mistrust may be particularly salient to those with presumably greater degrees of marginalization (i.e., ethnic minority vs. White MSM).

To our knowledge, no study to date has utilized a longitudinal model to examine the impact of HIV/AIDS-related institutional mistrust on HIV testing and sexual risk behavior. Longitudinal designs provide much stronger evidence for causal associations, particularly in the realm of sexual risk behavior where individuals might alter their attitudes and beliefs post-hoc to be consistent with or justify past risk behavior (Huebner, Neilands, Rebchook, \& Kegeles, 2011). And, as indicated by Bogart \& Bird (2005), more longitudinal data are needed to better distinguish whether HIV-related mistrust influences behavior, whether risk behavior influences mistrust, or if both processes are supported.

The current study will build upon the emerging empirical literature suggesting that mistrust fosters negative attitudes and suspicion toward prescribed preventive practices (Bogart \& Thorburn, 2005). Thus, we expect that men with higher levels of institutional mistrust will exhibit increased likelihood of engagement in sexual risk behavior and will be less likely to have been tested for HIV. To test the possibility that dual oppression affects these relationships, we will examine ethnic minority status as a possible moderator of the effects of institutional mistrust. We expect the hypothesized relationships to be more pronounced among ethnic minority MSM. To consider the question of "how" mistrust might influence sexual risk or testing behavior we will draw upon theories of health behavior (e.g., Witte, 1992) to explore the possibility that perceived susceptibility to HIV acts as a mediating cognitive process between mistrust and HIV-related health behaviors.

\section{Methods}

\section{Procedures and Participants}

Data were collected as a component of the "Heads Up Project," an initiative to examine the experiences of MSM with a focus on awareness, use, and opinions of local HIV prevention services in Central Arizona. A goal of the project was to recruit a sample of men who were diverse with respect to age, ethnicity, education, income, HIV-status, intravenous drug use, and residence (i.e., urban vs. rural). The project entailed 3 phases, including extensive pilot testing and focus groups, initial data collection (T1), and a 6-month follow-up (T2). Trust/mistrust of HIV-related institutions emerged from the initial pilot phase as a relevant issue to explore in subsequent phases. Details of the recruitment strategies and methods have been reported elsewhere (e.g., Hoyt, Nemeroff, \& Huebner, 2006; Huebner, Nemeroff, \& Davis, 2005). The Arizona State University Institutional Review Board and the Arizona Department of Health Services' Ethics Board approved all research methods.

Participants were males who identified as gay, bisexual, or "two-spirited" (for Native American participants) or reported sexual contact with another man in the past year; were at least 18 years of age; and had primary residence in the surrounding metropolitan area and neighboring rural counties. All MSM in a 3-county region were eligible. Six hundred and forty-seven men agreed to participate in the longitudinal study and were mailed a follow-up questionnaire at T2. Of these, 399 (62\%) completed the 
follow-up survey. Reasons for attrition included (a) those to which a follow-up questionnaire could not be sent either because they did not provide valid contact information or because they moved without providing a forwarding address, and (b) those who received a questionnaire and chose not to respond. Responders and non-responders did not differ significantly in their rates of sexual risk behavior, perceived susceptibility to HIV, level of institutional mistrust, or education. However, responders were older ( $M$ age $=37$ years vs. 33 years), wealthier, and more "out" than non-responders. Additionally, African American men were less likely to be responders to the follow-up survey; no other ethnic differences were found. Given the study objectives, participants who did not identify their ethnic identity $(n=5)$ were excluded, resulting in a final sample size of 394 men. Those who did not report ethnic identity did not differ on other demographic characteristics, level of institutional mistrust, or dependent variables. All available data were used for each analysis, and differences in sample sizes for each analysis are due to missing data. Men with missing data did not differ from men with complete data in any analysis in terms of demographic characteristics, level of institutional mistrust, or dependent variables.

Seventy-six percent of the participants were White, 13\% Latino, 5\% African American, 4\% Native American, and 2\% Asian American. Relative to the population of central Arizona, Native American and African American participants were over-represented in this sample due to intentional oversampling, while Latino participants were slightly under-represented. In this study, 'ethnic minority' was defined as any non-White participant. The mean age was 37 years $(S D=11.35)$. Nearly $42 \%$ of participants had earned a college degree or above, and the modal personal income fell between $\$ 25,000$ and $\$ 35,000$. Eight percent of the sample identified as "bisexual" and $90 \%$ self-identified as "gay." The remaining $2 \%$ identified as "straight" $(n=4)$, "two-spirited" $(n=2)$, or "unsure" $(n=4)$. Two men did not respond to this question.

\section{Measures}

Participants self-reported their age and other demographic information, including ethnicity. In addition, the following measures were obtained:

\section{Predictors of HIV-related health behaviors.}

We developed an 11-item Institutional Mistrust Scale to measure the degree of mistrust of HIV/AIDS-related institutions (e.g., medical services, government), which was administered at T1. Items were generated to represent themes that emerged from existing literature and pilot phase interviews and focus groups. Each item was measured on a 5-point scale ranging from responses reflecting low (1) to high (5) levels of mistrust. Using Mplus (Muthén \& Muthén, 2004), confirmatory factor analysis supported 3 dimensions of institutional mistrust that were determined a priori $\left[\chi^{2}(41)=60.96, p=.011\right.$; $\mathrm{CFI}=$.99; RMSEA = .039; SRMR = .045]. These included: Systematic Discrimination (4 items, e.g., "How much discrimination do you think there has been in access to HIV-related prevention services?"; "How much discrimination do you think there has been in policies concerning HIV/AIDS?"); Organizational Suspicion (4 items, e.g., "How much would you trust an HIV prevention organization that is run by the county or state government?"); and Conspiracy Beliefs (3 items, e.g., "How likely is it that AIDS was developed as part of a government plan?"; "How likely is it that certain minority groups have been exploited as medical guinea pigs in the treatment of HIV/AIDS?"). Factor loadings for individual items 
ranged from .75 to .85 for Systematic Discrimination, .38 to .93 for Organizational Suspicion, and .54 to .76 for Conspiracy Beliefs. Correlations with a total scale score were .75, .60, and .61 for Systematic Discrimination, Organizational Suspicion, and Conspiracy Beliefs, respectively. Cronbach's alphas for the Systematic Discrimination, Organizational Suspicion, and Conspiracy Beliefs sub-scales were $.86, .77$, and .76 , respectively. Responses were averaged to create 3 subscale scores which ranged from 0 to 5 .

Perceived susceptibility to HIV

This was measured at T1 with 3 items drawn from previous research (Aspinwall, Kemeny, Taylor, Schneider, \& Dudley, 1991; Bryan, Aiken, \& West, 1997; Gerrard, Gibbons, \& Bushman, 1996): "When you compare yourself to the average gay/bisexual man, how likely are you to get HIV?", "Would you say that you are the type of person who is likely to get HIV?", and "Considering all of the different factors that may contribute to HIV, including your own past and present behavior, how likely would you be to get HIV?" Responses were on a five-point Likert scale. In this sample Cronbach's alpha=.84.

HIV-related health behaviors.

Sexual risk behavior was defined as any unprotected receptive or insertive anal intercourse with any casual or steady male partner and was assessed at T1 and T2. By this definition, $60 \%$ of the sample ( $56 \%$ of White MSM; $70 \%$ of ethnic minority MSM) had engaged in sexual risk behavior in the 6 months prior to study entry; at T2 $56 \%$ of the sample (57\% of White MSM; $55 \%$ of ethnic minority MSM) had engaged in sexual risk behavior in the past 6 months.

Participants self-reported whether they had been tested for HIV in the past. Eighty-four percent of the sample (87\% of White MSM; $77 \%$ of ethnic minority MSM) reported having ever been tested for HIV.

\section{Results}

\section{Characteristics of Mistrust and Selection of Covariates}

Means and standard deviations of primary study variables were examined for the full sample as well as separately for ethnic minority and White MSM (see Table 1). Among these, only group differences on endorsement of Conspiracy Beliefs were statistically significant $(p=.002)$, with ethnic minority participants endorsing greater conspiracy beliefs. Intercorrelations of primary study variables are reported for White and ethnic minority MSM in Table 2. To identify the influence of sociodemographic factors, age, education, and income were entered as a single block in a regression model predicting dependent variables in separate regressions. Of these, only age was significantly associated with sexual risk behavior $(r=-.18, p<.01)$. Therefore, age was retained as a control variable in subsequent analyses.

\section{Mistrust and Sexual Risk Behavior}

Multiple logistic regression analysis was used to determine the degree to which the institutional mistrust subscales at T1 predicted sexual risk behaviors at T2. Analyses controlled for the autoregressive effects of T1 sexual risk behavior, as well as age. Thus, T2 risk behavior was regressed on T1 risk 
behavior, participant age, ethnic minority status (ethnic minority MSM vs. White MSM), the respective mistrust dimension, and the interactions of minority status and dimensions of mistrust. Results are reported in Table 3. No significant main effects of any mistrust dimension or minority status were observed on T2 sexual risk behaviors. However, the interactions of Systematic Discrimination and Conspiracy Beliefs with minority status were significant. In each case, regression lines were computed for ethnic minority and White MSM. Neither Systematic Discrimination ( $B=.14, n s)$ nor Conspiracy Beliefs $(B=.03, n s)$ were significantly related to sexual risk behavior for White MSM; however, both were significantly related to risk behavior for ethnic minority MSM. Higher levels of Systematic Discrimination $[B=.64, p<.05 ;$ OR $=1.90$ (95\% Cl: .98-2.68)] and more Conspiracy Beliefs $[B=.91, p<$ $.05 ; \mathrm{OR}=.40(95 \% \mathrm{Cl}: .19-.86)]$ were related to increased likelihood of engagement in sexual risk behavior among ethnic minority MSM.

To explore the possibility that Systematic Discrimination and/or Conspiracy Beliefs affected risk behavior differently among groups, individual regressions were conducted post-hoc for Latino, African American, and Native American MSM. This was not done for Asian American MSM due to their small number in the sample. Due to sample size constraints, none of these post-hoc tests were powered to detect statistically significant associations; despite this, inspection of the magnitudes of effects did not reveal that any one ethnic group was driving the overall effects of ethnicity observed in the analyses above.

\section{Mistrust and HIV Testing}

Next, similar analyses were conducted to examine relationships with HIV testing. HIV testing status (ever tested vs. never tested) was regressed on risk behavior, ethnic minority status (ethnic minority MSM vs. White MSM), the respective mistrust dimension, and the interactions of minority status and dimensions of mistrust. Sexual risk behavior was included in the model to control for other motivations for testing. Results are reported in Table 4. Higher levels of Systematic Discrimination were related to lower likelihood of having received HIV testing $(B=-.48, p<.01)$. Also, the interaction of Organizational Suspicion with minority status was significant. Regression lines were computed for ethnic minority and White MSM. Organizational Suspicion was not significantly related to HIV testing for White MSM ( $B=.01, n s)$; however, a significant relationship was found for ethnic minority MSM such that greater levels of Organizational Suspicion predicted less likelihood of having been tested for HIV $(B=-$ $.97, p<.05)$.

Individual regressions were conducted post-hoc for Latino, African American, and Native American MSM to explore the possibility that Organizational Suspicion was differently associated with HIV testing among the different groups. Although no significant relationships were found, Organization Suspicion approached significance among Hispanic MSM (B =-1.29, $p=.08)$. Again, due to sample size constraints, none of these post-hoc tests were powered to detect statistically significant associations.

\section{Mediation Analyses for Perceived Susceptibility}

Next, Baron and Kenny's (1986) method was adopted to consider the mediational hypothesis related to perceived susceptibility. An essential condition for mediation is that the main predictor (i.e., mistrust) must be associated with the mediating variable (i.e., perceived susceptibility) and the outcomes (i.e., HIV testing, sexual risk behavior). Based on the results of analyses above (see Tables 3 
and 4), three candidates for mediation emerged: (1) the association of Organizational Suspicion with HIV testing for ethnic minority MSM; (2) the association of Systematic Discrimination with sexual risk behavior for ethnic minority MSM; and (3) the association of Conspiracy Beliefs with sexual risk behavior for ethnic minority MSM. However, as shown in Table 2, none of the three subscales were significantly associated with perceived susceptibility for ethnic minority MSM (Organizational Suspicion, $r=-.08, n s$; Systematic Discrimination $r=-.15$, ns; Conspiracy Beliefs, $r=-.04, n s)$. Thus, no support was found for perceived susceptibility as a mediator of the relationship between institutional mistrust, sexual risk behavior, and HIV testing. Notably, detection of relationships was underpowered $(<.50$; as determined using G*Power, Version 3.1.2; Faul, 2009).

\section{Discussion}

Much research has documented health disparities between groups in the U.S., including disparities in utilization of health care and prevention services (e.g., Partnership for Prevention, 2007). Mistrust of health care organizations and health professionals may be one cultural factor contributing to these health disparities. The current findings highlight the importance of beliefs about HIV-related institutions and disease-origins to prevention efforts. The results of this study support our two key hypotheses: first, institutional mistrust is not a unitary construct. We identify organizational suspicion, conspiracy beliefs, and systematic discrimination as three facets of institutional mistrust. Second, various forms of institutional mistrust might differentially affect HIV-related risk and protective behaviors across groups. Perceptions of Systematic Discrimination was related to lower likelihood of having received HIV testing across groups, perhaps suggesting that participants were concerned about experiencing HIV-related discrimination either during testing, or based on their testing results. In contrast, and specifically among ethnic minority MSM, Conspiracy Beliefs, such as those related to the origins of the virus, may particularly impact behaviors associated with risk for contracting HIV; whereas Organizational Suspicion may affect trust and comfort interacting with HIV-related services, including organizations that provide HIV testing.

Importantly, our findings show that higher levels of the three measured dimensions of mistrust in HIV-prevention organizations and health care institutions are particularly detrimental to ethnic minority MSM, leading to increased likelihood of sexual risk behavior and lowered likelihood of HIV testing. It stands to reason that institutional mistrust would pose a barrier to service utilization, given that use of services such as HIV testing requires not only interacting with, but also accepting intrusive interventions which might be construed as dangerous from, entities that one does not trust. However, mistrust also appears to interfere with acceptance of prevention messages more broadly, as indicated by increased likelihood of sexual risk behaviors with increasing levels of mistrust. Although it was expected that the various dimensions of HIV-related institutional mistrust would predict increased odds of sexual risk behavior over time, no main effects for any form of mistrust were observed. The significant interaction effects highlight the manner in which ethnic minority MSM may be particularly vulnerable to negative consequences of institutional mistrust. Both Systematic Discrimination and Conspiracy Beliefs were associated with increased likelihood of sexual risk behavior among ethnic minorities, consistent with previous studies (e.g., Bogart \& Bird, 2003; Bogart \& Thorburn, 2005). Systematic Discrimination was associated with lower likelihood of receiving HIV testing across all ethnic 
groups. However, interaction effects revealed that suspicion in HIV-related organizations is particularly relevant to HIV testing likelihood for ethnic minority MSM.

It is likely that institutional mistrust arises out of specific motivations and cultural histories that are disparate across groups, and so they may be experienced quite differently. Therefore, such beliefs might be associated with feelings of particular vulnerability to HIV for some, but to a disregard for risk among others. Further, it is likely that the specific content of the mistrust influences perceptions of susceptibility and attitudes towards prevention differently. For instance, Bogart and colleagues (e.g., Bogart \& Bird, 2003; Bogart \& Thorburn, 2005) found different associations of conspiracy beliefs to condom-related attitudes and behaviors depending on the nature of the beliefs in samples of African Americans. Treatment-related conspiracy beliefs (e.g., antiretroviral medications are toxic) were related to more positive attitudes toward condoms, while government-related conspiracy beliefs (e.g., HIV was created in a government laboratory) had opposite relationships. Thus, the nature of the belief might affect what one believes about the HIV virus and its transmission, while other beliefs might be more apt to affect trust in medical testing and treatment services. The current study did not distinguish at this specific level in its measurement of conspiracy beliefs, looking instead at three broad but distinct dimensions of mistrust.

No support was found for the hypothesis that perceived susceptibility to HIV mediates mistrust and HIV testing or sexual risk behavior. In fact, only a moderate correlation with Conspiracy Beliefs was found with perceptions of susceptibility for White MSM. It may be that more specificity is needed in the identification of perceptions of vulnerability (e.g., perceived susceptibility to contracting HIV through behavior vs. feeling vulnerable to malicious, intentional infection) to better distinguish these relationships, or that a true relationship does not exist. It may be that the psychological states of trust and mistrust have a complex relationship with perceptions of susceptibility. For instance, entrusting one's health or wellbeing to an institution might also involve feelings of uncertainty and vulnerability to some extent (see Kramer, 1999). Notably, mediation analyses are notoriously underpowered in most studies, particularly when outcomes are dichotomous (MacKinnon, Lockwood, Hoffman, West, \& Sheets, 2002). More studies with larger samples of ethnic minority MSM are needed to elucidate potential mediating processes. Further, additional mediators should be considered. For instance, some aspects of mistrust may relate to a disruption in self-regulatory processes, which have been linked to riskier sexual behavior (Wiederman, 2004). Processes of impulsivity or emotion regulation may be particularly relevant. In fact, specific affective states (e.g., fear, hopelessness) might be implicated in a cascade of behavioral responses. Finally, socio-cultural contextual factors may potentially be fruitful mediators as well (e.g., experiences of racism, exposure to discriminative practices, health-related beliefs).

\section{Study Limitations}

A significant strength of the current study was to examine the effects of various dimension of mistrust on changes in risk over time. At the same time, several limitations should be acknowledged when considering these results. Although this study considers the intersections of sexual and ethnic minority identities, we did not have sufficient power to adequately examine multiple between group differences. Despite the fact that aspects of mistrust have been shown to be higher across ethnic minority groups (e.g., Hutchinson et al., 2007), future work should examine ethnic group differences, as well as possible interactions between ethnic identity and sexual orientation in primary analyses. 
Limitations to the generalizability of results should also be noted. Although significant efforts were employed to ensure representativeness of the sample, participants were not randomly sampled and a relatively small sample of multi-ethnic MSM was obtained. Finally, this study relied on a new measure of institutional mistrust. Although scale properties were good in this study, scale validation will improve with its utilization in additional studies with diverse samples.

\section{Implications and Future Directions}

Understanding the impact of mistrust within marginalized groups has several clinical and practice-related implications that should be considered. In addition to a resource or service-based orientation, models of trust and mistrust in institutions also emphasize a social or relational orientation (see Kramer, 1999). Understanding the generation of trust, and perhaps the mitigation of mistrust, as resulting from the social context supports the importance of the development and strengthening of minority organized and run AIDS Service Organizations. Health promotion efforts from such organizations may be less susceptible to mistrust by its constituents. In fact, HIV prevention efforts developed through culturally appropriate participatory leadership processes within ethnic minority communities appear to reduce institutional mistrust and foster civic engagement in further prevention efforts (e.g., Metayer, Jean-Louis, \& Madison, 2004). Minority-run AIDS service organization led interventions may also be more likely to foster engagement in clinical service and research (e.g., Gwadz et al., 2010). Relational processes of institutional trust building may also be modeled by civic and political leaders (Zimmer, 1972). Thus, government services, such as local health departments, that deliver more culturally competent service or employ more ethnic minority outreach workers may be more effective in reducing elements of institutional mistrust. Likewise, HIV research led and conducted by minority researchers or initiatives that utilize methods of community-engaged scholarship may be better positioned to invoke trust (see Christopher, Watts, McCormick, \& Young, 2008). Finally, Cialdini (1996) has discussed how surveillance can erode trust by engendering feelings of suspicion toward the surveyor and thus undermining motivation to engage in intended behaviors. Continued governmentbased HIV/AIDS surveillance reports and media attention that highlight infection and risk behavior among marginalized groups might foster mistrust and result in an ironic effect on protective and riskrelated health behaviors. More attention should be given to crafting health communications aimed at presenting data and identifying populations at high risk.

On the individual level, the development of mistrust of the medical community and associated institutions likely serves as a coping response in the face of threats of racism and discrimination that have historically impacted sexual and ethnic minority groups seeking medical care. However, different individual and community level experiences with medical institutions may result in different coping responses among White and ethnic minority MSM. For instance, mistrust in the form of suspicion in HIVrelated organizations was associated with lowered likelihood of being tested for HIV only among ethnic minority men in this study. Although ethnic minority MSM did not report significantly higher levels of Organizational Suspicion than White MSM in the current study, viewing medical and government organizations with some suspicion may differently affect health care utilization. Organizational Suspicion may foster approach-oriented responses (e.g., garnering multiple medical opinions, information-seeking from multiple sources) for some and avoidance behavior (e.g., reluctance to seek care) for others. 
Increasing the availability of acceptable approach-oriented responses (e.g., access to home HIV testing kits) may be one way to foster more adaptive responses to Organizational Suspicion.

Avoidant coping responses, particularly those associated with greater Organization Suspicion, likely place minority men at greater risk for under-utilization of medical services (Whaley, 2004), or contributes to behavior that insulates them from exposure to prevention messages or services (e.g., social withdrawal). Broad efforts to prevent HIV and other sexually transmitted infections require consideration of potential culturally-specific coping practices and culturally-related mistrust of specific aspects of health communication and education, which may become particularly salient in efforts to develop and distribute vaccines and other preventative medical interventions.

In sum, future work should assess mistrust as a multidimensional construct, and further elucidate misconceptions and culturally promulgated myths about HIV, reinforcements of mistrust beliefs, and the relationships of discrimination and marginalization to institutional mistrust. Further, aspects of trust have been identified as a personal resource of socially resilient individuals (Cacioppo, Reis, \& Zautra, 2011). Thus, clinical interventions that foster an ability to critically trust health-related institutions and information should be developed and tested. To the extent that this may require actually altering aspects of service delivery and habitual institutional practices, such possibilities should be considered and developed as well. 


\section{References}

Aspinwall, L.G., Kemeny, M.E., Taylor, S.E., Schneider, S.G., \& Dudley, J.P. (1991). Psychosocial predictors of gay men's AIDS risk-reduction behavior. Health Psychology, 10, 432-444.

Baron, R., \& Kenny, D. (1986). The moderator-mediator variable distinction in social psychological research: Conceptual, strategic, and statistical considerations. Journal of Personality and Social Psychology, 51, 1173-1182.

Bird, S.T., \& Bogart, L.M. (2005). Conspiracy beliefs about HIV/AIDS and birth control among African Americans: Implications for the prevention of HIV, other STI's, and unintended pregnancy. Journal of Social Issues, 61, 109-126.

Bogart, L.M., \& Bird, S.T. (2003). Exploring the relationship of conspiracy beliefs about HIV/AIDS to sexual behaviors and attitudes among African American adults. Journal of the National Medical Association, 95, 1057-1065.

Bogart, L.M., Galvan, F.H., Wagner, G.J., \& Klein, D.J. (2010). Longitudinal association of HIV conspiracy beliefs with sexual risk among Black males living with HIV. AIDS \& Behavior.

Bogart, L.M., \& Thorburn, S. (2005). Are HIV/AIDS conspiracy beliefs a barrier to HIV prevention among African Americans? Journal of Acquired Immune Deficiency Syndrome, 38, 213-218.

Bogart, L.M., Wagner, G., Galvan, F.H., \& Banks, D. (2010). Conspiracy beliefs about HIV are related to antiretroviral treatment nonadherence among African American men with HIV. Journal of Acquired Immune Deficiency Syndromes, 53, 648-655.

Bowleg, L., Huang, J., Brooks, K., Black, A., \& Burkholder, G. (2003). Triple jeopardy and beyond: Multiple minority stress and resilience among Black lesbians. Journal of Lesbian Studies, 7, 87-108.

Brandon, D.T., Isaac, L.A., \& LaVeist, T.A. (2005). The legacy of Tuskegee and trust in medical care: Is Tuskegee responsible for race differences in mistrust of medical care? Journal of the National Medical Association, 97, 951-956.

Bryan, A.D., Aiken, L.S., \& West, S.G. (1997). Young women's condom use: The influence of acceptance of sexuality, control over the sexual encounter, and perceived susceptibility to common STDs. Health Psychology, 16, 468-479.

Cacioppo, J.T., Reis, H.T., \& Zautra, A.J. (2011). Social resilience: The value of social fitness with an application to the military. American Psychologist, 66, 43-51.

CDC. (2010, September). HIV and AIDS among gay and bisexual men. Retrieved from http://cdc.gov/nchhstp/newsroom/docs/FastFacts-MSM-FINAL508COMP.pdf

CDC. (2007). Racial/ethnic disparities in diagnoses of HIV/AIDS - 33 states, 2001-2005. MMWR, 56, 189193.

Christopher, S., Watts, V., McCormick, A.K., \& Young, S. (2008). Building and maintaining trust in a community-based participatory research partnership. American Journal of Public Health, 98, 1398-1406.

Cialdini, R. (1996). The triple tumor structure of organizational behavior. In D.M. Messick \& A.E. Tenbrunsel (Eds.), Codes of conduct (pp. 44-58). New York: Russell Sage Foundation.

Dean, L., Meyer, I.H., Robinson, K., Sell, R.L., Sember, R., Silenzio, V.M.B., ... Xavier, J. (2000). Lesbian, gay, bisexual, and transgender health: Findings and concerns. Journal of the Gay and Lesbian 
Medical Association, 4, 101-151. Faul, F. (2009). G*Power (Version 3.1.2) [Computer software]. Kiel, Germany: Universität Kiel.

Gamble, V.N. (1997). Under the shadow of Tuskegee: African Americans and health care. American Journal of Public Health, 87, 1773-1778.

Gerrard, M., Gibbons, F.X., \& Bushman, B.J. (1996). Relation between perceived vulnerability to HIV and precautionary sexual behavior. Psychological Bulletin, 19, 390-409.

Gutiérrez, E.R. (2008). Fertile matters: The politics of Mexican-origin women's reproduction. Austin, TX: University of Texas Press.

Gwadz, M.V., Cylar, K., Leonard, N.R., Riedel, M., Herzog, N., Arredondo, G.N., Cleland, C.M., Aquirre, M., Marshak, A., \& Mildvan, D. (2010). An exploratory behavioral intervention trial to improve rates of screening for AIDS clinical trials among racial/ethnic minority and female persons living with HIV/AIDS. AIDS and Behavior, 14, 639-648.

Halbert, C.H., Weathers, B., Delmoor, E., Mahler, B., Coyne, J., Thompson, H.S., .... Lee, D. (2009). Racial differences in medical mistrust among men diagnosed with prostate cancer. Cancer, 115, 25532561.

Hammond, W.P. (2010). Psychosocial correlates of medical mistrust among African American men. American Journal of Community Psychology, 45, 87-106.

Herek, G.M., \& Capitanio, J.P. (1994). Conspiracies, contagion, and compassion: Trust and public reactions to AIDS. AIDS Education and Prevention, 6, 365-375.

Hoyt, M.A., Nemeroff, C.J., \& Huebner, D.M. (2006). The effects of HIV-related thought suppression on risk behavior: Cognitive escape in men who have sex with men. Health Psychology, 25, 455-461.

Huebner, D.M., Neilands, T.B., Rebchook, G.M., \& Kegeles, S.M. (2011). Sorting through chickens and eggs: A longitudinal examination of the associations between attitudes, norms, and sexual risk behavior. Health Psychology, 30, 110-118.

Huebner, D. M., Nemeroff, C. J., \& Davis, M. C. (2005). How much do hostility and neuroticism confound associations between perceived discrimination and depressive symptoms? Journal of Social and Clinical Psychology, 24, 723-740.

Hutchinson, A.B., Begley, E.B., Sullivan, P., Clark, H.A., Boyett, B.C., \& Kellerman, S.E. (2007). Conspiracy beliefs and trust in information about HIV/AIDS among minority men who have sex with men. Journal of Acquired Immune Deficiency Syndrome, 45, 603-605.

Jennings, M.K., \& Andersen, E.A. (2003). The importance of social and political context: The case of AIDS activism. Political Behavior, 25, 177-199.

Kalichman, S. (2009). Denying AIDS: Conspiracy theories, pseudoscience, and human tragedy. New York, NY: Springer.

Klonoff, E.A., \& Landrine, H. (1999). Do Blacks believe that HIV/AIDS is a government conspiracy against them? Preventive Medicine, 28, 451-457.

Kramer, R.M. (1999). Trust and distrust in organizations: Perspectives, enduring questions. Annual Review of Psychology, 50, 569-598.

López, I. (2008). Matters of choice: Puerto Rican women's struggle for reproductive freedom. New Brunswick: Rutgers University Press. 
MacKinnon, D.P., Lockwood, C.M., Hoffman, J.M., West, S.G., \& Sheets, V. (2002). A comparison of methods to test mediation and other intervening variable effects. Psychological Methods, 7, 83104.

Malebranche, D.J., Peterson, J.L., Fullilove, R.E., \& Stackhouse, R.W. (2004). Race and sexual identity: Perceptions about medical culture and healthcare among Black men who have sex with men. Journal of the National Medical Association, 96, 97-107.

Magnus, M., Kuo, I., Phillips, G., Shelley, K., Rawls, A., Montanez, L., Peterson, J., West-Ojo, T., Hader, S., \& Greenberg, A.E. (2010). Elevated HIV prevalence despite lower rates of sexual risk behaviors among Black men in the District of Columbia who have sex with men. AIDS Patient Care and STDs, 24, 615-622.

Metayer, N., Jean-Louis, E., \& Madison, A. (2004). Overcoming historical and institutional distrust: Key elements in developing and sustaining the community mobilization against HIV in the Boston Haitian community. Ethnicity and Disease, 14, S46-S52.

Millett, G.A., Peterson, J.L., Wolitski, R.J., \& Stall, R. (2006). Greater risk for HIV infection of Black men who have sex with men: A critical literature review. American Journal of Public Health, 96, 10071019.

Mullen, P.D., Hersey, J., \& Iverson, D.C. (1987). Health behavior models compared. Social Science and Medicine, 24, 973-981.

Musa, D., Schulz, R., Harris, R., Silverman, M., \& Thomas, S.B. (2009). Trust in the health care system and the use of preventive health services by older Black and White adults. American Journal of Public Health, 99, 1293-1299.

Muthén, L.K., \& Muthén, B.O. (2004). Mplus (Version 3.0) [Computer software]. Los Angeles, CA: Muthen \& Muthen.

Omodei, M.M., \& McLennan, J. (2000). Conceptualizing and measuring global interpersonal mistrusttrust. Journal of Social Psychology, 140, 279-294.

Owens, G.P., Riggle, E.D.B., \& Rostosky, S.S. (2007). Mental health services access for sexual minority individuals. Sexuality Research \& Social Policy: A Journal of the NSRC, 4(3), 92-99.

Parsons, S., Simmons, W., Shinhoster, F., \& Kilburn, J. (1999). A test of the grapevine: An empirical examination of conspiracy theories among African Americans. Sociological Spectrum, 19, 201222.

Partnership for Prevention. (2007). Preventive care: A national profile on use, disparities, and health benefits. Washington, DC: Partnership for Prevention. Retrieved from http://www.rwjf.org/files/research/8-7-07\%20-

\%20Partnership\%20for\%20Prevention\%20Report(1).pdf

Roberts, D. (1997). Killing the Black body. New York: Random House.

Ross, M.W., Essien, E.J., \& Torres, I. (2006). Conspiracy beliefs about the origins of HIV/AIDS in four racial/ethnic groups. Journal of Acquired Immune Deficiency Syndromes, 41, 342-344.

Scharff, D.P., Mathews, K.J., Hoffsuemmer, J., Martin, E., \& Edwards, D. (2010). More than Tuskegee: Understanding mistrust about research participation. Journal of Health Care for the Poor and Underserved, 21, 879-897.

Sheran, M. (1995). The challenge of treating gay men with AIDS. Bulletin of the New York Academy of Medicine, 72, 202-210. 
Smedley, B.D., Stih, A.Y., \& Nelson, A.R. (Eds.) (2003). Unequal treatment: Confronting racial and ethnic disparities in health care. Washington, DC: The National Academies Press.

Thomas, S.B., \& Quinn, S.C. (1993). The burdens of race and history on Black Americans attitudes toward needle exchange policy to prevent HIV disease. Journal of Public Health Policy, 14, 320-347.

Thompson, H.S., Valdimarsdottir, H.B., Winkel, G., Jandorf, L., \& Redd, W. (2004). The group-based medical mistrust scale: Psychometric properties and association with breast cancer screening. Preventive Medicine: An International Journal Devoted to Practice and Theory, 38, 209-218.

Vernon, I.S., \& Jumper-Thurman, P. (2002). Prevention of HIV/AIDS in Native American communities: Promising interventions. Public Health Reports, 117, S96-S103.

Whaley, A.L. (2004). Ethnicity/race, paranoia, and hospitalization for mental health problems among men. American Journal of Public Health, 94, 78-81.

Whetten, K., Reif, S., Whetten, R., \& Murphy-McMillan, K. (2008). Trauma, mental health, distrust, and stigma among HIV-positive persons: Implications for effective care. Psychosomatic Medicine, 72, 531-538.

Wiederman, M.W. (2004). Self-control and sexual behavior. In R.F. Baumeister \& K.D. Vohs (Eds.), Handbook of self-regulation: Research, theory, and applications. (pp. 525-536). New York: Guilford Press.

Witte, K. (1992). Putting the fear back into fear appeals: The extended parallel process model. Communication Monographs, 59, 330-349.

Zimmer, T. (1972). The impact of Watergate on the public's trust in people and confidence in the mass media. Social Science Quarterly, 59, 743-751. 
Table 1

Means and Standard Deviations of Study Variables

\begin{tabular}{|c|c|c|c|c|c|c|}
\hline \multirow[b]{2}{*}{ Variable } & \multicolumn{2}{|c|}{$\begin{array}{l}\text { Total Sample } \\
\qquad(\mathrm{N}=394)\end{array}$} & \multicolumn{2}{|c|}{$\begin{array}{l}\text { Ethnic Minority MSM } \\
\qquad(\mathrm{n}=88)\end{array}$} & \multicolumn{2}{|c|}{$\begin{array}{l}\text { White MSM } \\
\qquad(\mathrm{n}=306)\end{array}$} \\
\hline & $\mathrm{M}$ & $\mathrm{SD}$ & $\mathrm{M}$ & $\mathrm{SD}$ & $\mathrm{M}$ & SD \\
\hline Organizational Suspicion & 2.46 & .85 & 2.58 & .83 & 2.43 & .85 \\
\hline Systematic Discrimination & 3.60 & 1.02 & 3.53 & .93 & 3.62 & 1.02 \\
\hline Conspiracy Beliefs & 1.97 & .89 & 2.23 & .93 & 1.89 & .86 \\
\hline
\end{tabular}


Table 2

Intercorrelations of Study Variables for White and Ethnic Minority MSM

Variable $\quad$ Ethnic Minority MSM $(\mathrm{n}=88)$

White MSM ( $\mathrm{n}=306)$

\begin{tabular}{|c|c|c|c|c|c|c|c|c|c|c|c|c|c|c|}
\hline & 1 & 2 & 3 & 4 & 5 & 6 & 7 & 1 & 2 & 3 & 4 & 5 & 6 & 7 \\
\hline 2. T2 Sexual Risk & $.40 * * *$ & - & & & & & & $.56 * * *$ & - & & & & & \\
\hline 3. HIV Testing & .18 & -.01 & - & & & & & $.18 * *$ & $.18 * *$ & - & & & & \\
\hline 4. Perceived Susceptibility & $.29 *$ & .12 & -.01 & - & & & & $.26 * * *$ & $.18^{* *}$ & .04 & - & & & \\
\hline \multicolumn{15}{|l|}{ to HIV } \\
\hline 6. Systematic & -.06 & -.08 & .03 & -.15 & -.01 & - & & .05 & $-.16^{*}$ & .08 & .05 & .06 & - & \\
\hline \multicolumn{15}{|l|}{ Discrimination } \\
\hline 7. Conspiracy Beliefs & .01 & -.18 & -.07 & -.04 & -.01 & $.32 * *$ & - & .03 & .01 & .01 & $.27 * * *$ & $.14^{*}$ & $.29 * * *$ & - \\
\hline
\end{tabular}

$* p<.05 ; * * p<.01 ; * * * p<.001$. 
Table 3

Logistic Regression Analyses Testing Moderating Effects of Ethnic Minority Status, Systematic Discrimination, Organizational Suspicion, and Conspiracy Beliefs on Sexual Risk Behavior

$\begin{array}{llllll}\text { Predictor Variable } & \text { B } & \text { SE B } & \text { Wald } \chi^{2}(1) & \text { OR }(95 \% \mathrm{CI}) & \Delta R^{2}\end{array}$

\section{Block 1}

Sexual risk behavior (Time 1)

2.50

Ethnic Minority Status

$\begin{array}{llll}.69 & .36 & 3.75 \$ & 2.00(.99-4.04)\end{array}$

Systematic Discrimination

$-.12 \quad .15$

.65

$.88(.66-1.19)$

Organizational Suspicion

$.22 \quad .17$

1.70

$1.25(.89-1.75)$

Conspiracy Beliefs

$.02 \quad .18$

.02

$1.02(.72-1.49)$

$\underline{\text { Block } 2}$

Systematic Discrimination X Ethnic Minority Status

.69

.35

$3.92 *$

$2.00(1.01-3.96)$

Organizational Suspicion X Ethnic Minority Status

$-.34 \quad .44$

.60

$.71(.30-1.69)$

Conspiracy Beliefs X Ethnic Minority Status

$-.93 \quad .40 \quad 5.49 *$

$.39(.18-.86)$

Note. Regression coefficients and Wald $\chi^{2}$ values reflect values at the end of Block 2, with all variables entered into the equation. OR $=$ Odds Ratio. $\mathrm{CI}=$ Confidence Interval. $\mathrm{SE}=$ Standard Error. Analyses controlled for age. $\$ p<.10 ; * p<.05 ; * * p<.01 ; * * * p<.001$. 
Table 4

Logistic Regression Analyses Testing Moderating Effects of Ethnic Minority Status, Systematic Discrimination, Organizational Suspicion, and Conspiracy Beliefs on HIV Testing

$\begin{array}{llllll}\text { Predictor Variable } & \text { B } & \text { SE B } & \text { Wald } \chi^{2}(1) & \text { OR }(95 \% \mathrm{CI}) & \Delta R^{2}\end{array}$

\section{Block 1}

Sexual Risk Behavior

Ethnic Minority Status

Systematic Discrimination

Organizational Suspicion

Conspiracy Beliefs

Block 2

Systematic Discrimination X Ethnic Minority Status

Organizational Suspicion X Ethnic Minority Status

Conspiracy Beliefs X Ethnic Minority Status

$\begin{array}{cccc}.97 & .32 & 9.19 * * & 2.63(1.41-4.90) \\ -.41 & .41 & 1.02 & .66(.30-1.47) \\ .48 & .18 & 7.17 * * & 1.61(1.14-2.28) \\ .01 & .21 & <.01 & 1.01(.67-1.52) \\ -.25 & .23 & 1.17 & .78(.50-1.22)\end{array}$

$\begin{array}{cccc}-.58 & .36 & 2.56 & .56(.28-1.14) \\ -.94 & .51 & 3.38 * & .49(.24-1.06) \\ -.20 & .45 & .20 & .82(.34-1.98)\end{array}$

.142

Note. Regression coefficients and Wald $\chi^{2}$ values reflect values at the end of Block 2 , with all variables entered into the equation. OR $=$ Odds Ratio. $\mathrm{CI}=$ Confidence Interval. $\mathrm{SE}=$ Standard Error. Analyses controlled for HIV status. $* p<.05 ; * * p<.01 ; * * * p<.001$. 